\title{
Nuclear magnetic resonance at millitesla fields using a zero-field spectrometer
}

\author{
Michael C. D. Tayler ${ }^{\mathrm{a}, \mathrm{b}, *}$, Tobias F. Sjolander ${ }^{\mathrm{c}}$, Alexander Pines ${ }^{\mathrm{c}}$, Dmitry \\ Budker $^{\mathrm{b}, \mathrm{d}}$ \\ ${ }^{a}$ Magnetic Resonance Research Center, Department of Chemical Engineering and \\ Biotechnology, University of Cambridge, Cambridge, UK. \\ ${ }^{b}$ Department of Physics, University of California, Berkeley, CA 94720, USA. \\ ${ }^{c}$ College of Chemistry, University of California Berkeley, CA 94720, USA. \\ ${ }^{d}$ Helmholtz Institut Mainz, Johannes Gutenberg University, Mainz 55099, Germany
}

\begin{abstract}
We describe new analytical capabilities for nuclear magnetic resonance (NMR) experiments in which signal detection is performed with chemical resolution (via spin-spin J couplings) in the zero to ultra-low magnetic field region, below $1 \mu \mathrm{T}$. Using magnetic fields in the $100 \mu \mathrm{T}$ to $1 \mathrm{mT}$ range, we demonstrate the implementation of conventional NMR pulse sequences with spin-species selectivity.
\end{abstract}

Keywords: Nuclear magnetic resonance, Ultra-low magnetic field, High-resolution NMR, Zero-field NMR, Atomic magnetometer, Pulse sequences

\section{Introduction}

Recently, NMR spectra have been measured with ultrahigh $(\approx 20 \mathrm{mHz})$ resolution in zero and ultralow magnetic field (ZULF).[1, 2, 3, 4, 5, 6, 7] This approach to NMR, instrumentally considerably less demanding than traditional high-field NMR with superconducting magnets, has the potential to be analytically useful for many of the experiments carried out on small to medium sized molecules. [8, 9] Examples of experiments on a variety of

\footnotetext{
${ }^{*}$ Corresponding author

Email address: mcdt2@cam.ac.uk (Michael C. D. Tayler)
} 
aromatic molecules demonstrated compelling spectral detail that differentiated between the compounds.[1] Zero-field spectroscopy is therefore a route to chemically informative NMR signals.

A ZULF NMR signal is recorded with the sample placed in a magnetically shielded environment where evolution of the spins is dominated by spinspin couplings, [10, 11, 12] (and sometimes nuclear quadrupole moments[13]), rather than Larmor precession. The absence of a magnetic field also avoids concerns of magnetic susceptibility and radiofrequency skin depth penetration, which may cause complications in high-field NMR spectroscopy.[14]. Following the initial pre-polarization or pre-hyperpolarization of the sample, the whole ZULF NMR experiment is carried out in situ by means of optical detection and without the need for sample shuttling.[15, 16, 17, 18, 19] As an alternative to previous ZULF NMR measurements using superconducting quantum interference devices, $[20,21,22,23,24,25]$ an inexpensive optical magnetometer obviates the need for cryogenics, adding to the potential mobility of the device.[26, 27]

While detection of NMR signals in ZULF has advantages, including ultranarrow linewidths, some features of high field are indispensable. In a highfield experiment, the strong static magnetic field ensures the wide separation of resonance frequencies for non-zero-spin nuclei (e.g. ${ }^{1} \mathrm{H},{ }^{13} \mathrm{C},{ }^{15} \mathrm{~N},{ }^{19} \mathrm{~F}$ ) allowing each to be addressed selectively using AC magnetic field pulses. Using pulses with much better frequency selectivity, individual spins with resolved, albeit with lower resolution chemical shifts $(\approx \mathrm{Hz})$ can be controlled. In ZULF, the selection of individual spin species is not straightforward, but can be achieved by means of complex pulse sequences due to the different precession frequencies of each spin species when a pulse is applied.[28, 29]

In this communication we report on the use of high-field selectivity in ZULF where a magnetic field is temporarily imposed on the sample, allowing one to apply $\mathrm{AC}$ pulses that individually address different spin species. Experiments performed on ${ }^{1} \mathrm{H}$ and ${ }^{13} \mathrm{C}$ in formic acid and on ${ }^{1} \mathrm{H}$ and ${ }^{19} \mathrm{~F}$ in difluoroacetic acid demonstrate pulse sequences performed at millitesla magnetic fields to flexibly vary the initial conditions for ZULF detection and the appearance of the associated spectra. The arsenal of well-established NMRbased methods for chemical analysis should add perceptibly to the fidelity of ZULF NMR and the spectroscopy of ultrahigh-resolution, chemically identified spin-spin couplings. 


\section{Experimental detail}

The experimental setup is based on a rubidium-87 $\left({ }^{87} \mathrm{Rb}\right)$ spin-exchangerelaxation-free (SERF) atomic magnetometer and is depicted in figure 1.[30] A ${ }^{87} \mathrm{Rb}$-vapor cell $\left(5 \times 5 \times 8 \mathrm{~mm}^{3}, 93 \mathrm{kPa} \mathrm{N}, 180{ }^{\circ} \mathrm{C}\right.$, Twinleaf LLC $)$ and the NMR sample (50-100 $\mu \mathrm{L}$ in a $5 \mathrm{~mm}$ o.d. glass tube) are surrounded by a multi-layer $\mu$-metal and ferrite shield, which suppresses background magnetic fields to a residual of less than $0.1 \mathrm{nT}$. As shown in figure 1, a set of three orthogonal electromagnetic coils is also placed in the magnetically shielded region to supply time-dependent magnetic fields for excitation of the spins and encoding of additional spectroscopic dimensions (three Helmholtz coils, each coil 28 gauge or $0.32 \mathrm{~mm}$ diameter enameled copper wire, 6 turns, radius $=2.2 \mathrm{~cm}$, magnetic field $=230 \mu \mathrm{T} / \mathrm{A})$. The magnetometer employs two laser beams (not drawn): one along the $x$ axis for optical pumping of the ${ }^{87} \mathrm{Rb}$ vapor and one along the $y$ axis serving to probe the atomic polarization. The measured signal is a voltage proportional to the component of magnetic flux at right angles to the two beams, along the $z$ axis.[17, 31, 32]

The experiment begins with a pre-polarization step, in our case by holding the sample in a strong magnetic field (2 T Halbach magnet: RHR-2T-10, Bflux Technology) outside the shielded zone to initialize a Zeeman-polarized state. The sample is then pneumatically shuttled into the ZULF region through a $9 \mathrm{~mm}$ i.d. fiberglass tube over $\approx 1 \mathrm{~s}$. In order to supply arbitrary magnetic field profiles, waveforms $( \pm 5 \mathrm{~V}, 16$ bit, amplitudes defined at 10 $\mu$ s time intervals) are programmed on a computer (using Wolfram Mathematica and LabView) and the controlled current is supplied to the respective coils using a microcontroller/DAQ card (National Instruments USB 6229), low-noise linear amplifiers (AE Techron LVC2016) and a switch (home-built, for schematic see figure 1) to isolate the magnetometer from electrical noise when pulsed fields are not being applied. The NMR signal is recorded digitally during an acquisition period and the spectrum obtained via a Fourier transform. Ignoring relaxation, the signal for a given molecule is given by the sum

$$
\operatorname{signal}(t) \propto \sum_{A, B}\left\langle A\left|\rho_{0}\right| B\right\rangle\left\langle A\left|M_{z}\right| B\right\rangle \exp \left(-\mathrm{i} \omega_{A B} t\right)
$$

where each $\omega_{A B}$ is the transition frequency between two eigenstates $|A\rangle$ and $|B\rangle$ of the nuclear spin Hamiltonian. For isotropic liquids in zero-field, this Hamiltonian $\left(H_{\mathrm{ZF}}\right)$ is assumed purely intramolecular, comprising the nuclear 
spin-spin $J$ couplings. The Fourier coefficients give the amplitude of each spectral line in terms of spin operators: $\rho_{0}$, representing the spin density matrix at the start of the measurement $(t=0)$ and $M_{z} \equiv \sum_{j} \gamma_{j} I_{z}^{j}$, the observable, namely the total magnetization along $z$, where $\gamma$ denotes the nuclear gyromagnetic ratio.

\section{Results and discussion}

The initial condition - the density operator $\rho_{0}$ - depends upon how the magnetic field varies from the prepolarizing field (the high field) to zero field. The equilibrium density operator in the prepolarization magnet is given approximately by $\rho_{\mathrm{eq}}=1+\left(\hbar B_{\mathrm{pol}} / k_{B} T\right) \sum_{j} \gamma_{j} I_{z}^{j}$, where $\hbar$ is Planck's constant divided by $2 \pi, B_{\text {pol }}$ is the field strength, $k_{B}$ is Boltzmann's constant and $T$ is the temperature. Two limiting cases of $\rho_{0}$ are of relevance: sudden and adiabatic switching of the field. In adiabatic switching, $\rho_{\text {eq }}$ is adiabatically transformed as the eigenstates of the spin system vary between weak coupling in high field and strong coupling at zero field.[2, 10] In sudden switching, the field is quenched on a timescale fast compared to the spin system eigenfrequencies, so that the density operator remains constant $\left(\rho_{0} \equiv \rho_{\text {eq }}\right)$.

Let us consider $\left[{ }^{13} \mathrm{C}\right]$-formic acid $\left(\mathrm{H}^{13} \mathrm{COOH}\right.$, Sigma Aldrich Isotec) as an example. Ignoring the labile proton, the nuclear spin system of the molecule contains one scalar coupled ${ }^{1} \mathrm{H}^{13} \mathrm{C}$ pair. For this system, $\rho_{\text {eq }}=$ $1+\left(\hbar B_{\mathrm{pol}} / k_{B} T\right)\left(\gamma_{\mathrm{H}} I_{z}^{\mathrm{H}}+\gamma_{\mathrm{H}} I_{z}^{\mathrm{C}}\right)$ and $H_{\mathrm{ZF}}=2 \pi^{1} J_{\mathrm{CH}}\left(I_{x}^{\mathrm{H}} I_{x}^{\mathrm{C}}+I_{y}^{\mathrm{H}} I_{y}^{\mathrm{C}}+I_{z}^{\mathrm{H}} I_{z}^{\mathrm{C}}\right)$ where ${ }^{1} J_{\mathrm{CH}}=222.1 \mathrm{~Hz}$ is the value of the one-bond ${ }^{1} \mathrm{H}-{ }^{13} \mathrm{C}$ scalar coupling. Note that the high-field equilibrium density operator $\rho_{\text {eq }}$ does not commute with $H_{\mathrm{ZF}}$ - a general result, in fact, for molecules containing two or more different spin species (a heteronuclear system). Consequently, in sudden switching, $\rho_{0}$ contains off-diagonal matrix elements that are nonzero yielding immediately an NMR signal without the need for an excitation pulse.

During adiabatic switching, the density operator must by definition vary smoothly and therefore on reaching zero-field be stationary under $H_{\mathrm{ZF}}$. A magnetic field pulse is required to excite a noncommuting state and produce a nonstatic NMR signal. The case of a square DC pulse of strength $B$ and duration $t_{\text {pulse }}=1.0 \mathrm{~ms}$ applied along the $z$ axis is illustrated in Figure $2 \mathrm{a}$ (pulse rise and fall times $\approx 10 \mu \mathrm{s}$ ). The plotted data represent the zero-field signal amplitude vs. $B$ and follow the form $\sin \left[\left(\gamma_{\mathrm{H}}-\gamma_{\mathrm{C}}\right) B t_{\text {pulse }}\right]$. This profile arises because the pulse rotates each spin about the field axis by an angle $\gamma_{i} B$. 
One could also use the pulse coils to reverse the adiabatic transformation induced by sample shuttling and return the spins to a "high field", weakly coupled regime with the spins oriented along the direction of the field. Figure $2 \mathrm{~b}$ shows the zero-field NMR signal of formic acid recorded after applying a smooth-rising pulse (hyperbolic secant: $B(t)=B \operatorname{sech}\left[15\left(t / \tau_{\text {pulse }}\right)\right] ;-\tau_{\text {pulse }} \leq$ $t \leq 0 ; \tau_{\text {pulse }}=100 \mathrm{~ms}$ ), followed by sudden quenching. The dependence of the signal amplitude (recorded from $t=0$ ) on $B$ is in this case non-oscillatory and approximately independent of the field strength in the high-field limit, $\left|\left(\gamma_{\mathrm{H}}-\gamma_{\mathrm{C}}\right) B\right| / 2 \pi>\left|J_{\mathrm{CH}}\right|$.

The adiabatic-remagnetization pulse naturally extends to the incorporation of conventional NMR pulse sequences in a zero-field experiment. We demonstrate this using the example of selective spin inversion. Such an example represents a selectivity that is taken for granted in almost all high-field NMR experiments where the pulse bandwidth is narrow in comparison to the resonance frequencies of the NMR-active nuclei, allowing for arbitrary manipulation of some nuclei while leaving others unperturbed.[33] In ZULF, where the spin system is fully coupled, this option is not available. Figure 3a shows an adiabatic transfer of spin order from zero field into a DC field of strength $B$ oriented along the $z$ axis. An AC field pulse (100 ms duration), typically weaker than $B$ by 1-2 orders of magnitude, is then applied using the $x$-axis coil. The amplitude of the AC pulse follows a half-sine profile while the frequency is linearly swept over a range of $\nu-\Delta \nu / 2$ to $\nu+\Delta \nu / 2$, where $\nu$ is the center frequency and $\Delta \nu$ is the sweep width. The effect of this pulse is to invert the $z$-axis magnetization component of the spins whose precession frequency is within the sweep range. [34, 35]

Simulated trajectories of the spin polarization operators for a ${ }^{1} \mathrm{H}_{-}{ }^{13} \mathrm{C}$ spin pair system are plotted in Figure 3b. A value $\Delta \nu=400 \mathrm{~Hz}$ is chosen to select only one spin species. The three different resonance conditions of the $\mathrm{AC}$ pulse are illustrated. The topmost plot (figure $3 \mathrm{~b}(\mathrm{i})$ ) is for the case where neither spin is on resonance and therefore is identical to the situation in Figure 2b. Ignoring spin relaxation, the density operator following the $\mathrm{DC}$ re-magnetization is equal to that in the pre-polarization field and the intensity of the zero-field NMR spectrum is proportional to the oscillatory component of $\rho_{0}$, in this case $\left(I_{z}^{\mathrm{H}}-I_{z}^{\mathrm{C}}\right) / 2$. The coefficient of this operator in $\rho_{\text {eq }}$ is $\left(\gamma_{\mathrm{H}}-\gamma_{\mathrm{C}}\right)$. The coefficient is changed when the pulse is resonant with one of the spin species. If the carbon spin is inverted, as in figure $3 \mathrm{~b}(\mathrm{ii})$, the density operator changes from $\rho_{0}=1+\left(\hbar B_{\mathrm{pol}} / k_{B} T\right)\left(\gamma_{\mathrm{H}} I_{z}^{\mathrm{H}}+\gamma_{\mathrm{C}} I_{z}^{\mathrm{C}}\right)$ to $\rho_{0}=1+\left(\hbar B_{\mathrm{pol}} / k_{B} T\right)\left(\gamma_{\mathrm{H}} I_{z}^{\mathrm{H}}-\gamma_{\mathrm{C}} I_{z}^{\mathrm{C}}\right)$, and the coefficient becomes $\left(\gamma_{\mathrm{H}}+\gamma_{\mathrm{C}}\right)$. 
If the proton is inverted instead (figure $3 \mathrm{~b}$ (iii)), there will be an additional negative sign: $\left(-\gamma_{\mathrm{H}}-\gamma_{\mathrm{C}}\right)$. These variations in signal amplitude are apparent in the red curves, which represent the trajectories of the total magnetization along the $z$ axis. The zero-field NMR signal is acquired starting at $1.0 \mathrm{~s}$.

Using the atomic magnetometer, the amplitude of the zero-field NMR signal for $\left[{ }^{13} \mathrm{C}\right]$-formic acid (spectral line at $\approx 222 \mathrm{~Hz}$ ) was measured vs. $B$ for different values of $\nu(2,4,8,16,32$ and $64 \mathrm{kHz})$, and the behavior is shown in Figure 4. As outlined above for the model ${ }^{1} \mathrm{H}_{-}{ }^{13} \mathrm{C}$ spin pair, resonances with the $\mathrm{AC}$ pulse manifest in positive and negative peaks where the signal amplitude changes by factors $\pm\left(\gamma_{\mathrm{H}}+\gamma_{\mathrm{H}}\right) /\left(\gamma_{\mathrm{H}}-\gamma_{\mathrm{C}}\right) \approx \pm 1.6$. The data agree with the expectation that there should be no perturbation on the nuclear spins outside the pulse bandwidth, namely the limits given in terms of field by $|B \pm \Delta B / 2|=|(\nu \pm \Delta \nu / 2) / \gamma|$ which are represented on the plots in Figure 4 as the shaded vertical bands $\left({ }^{1} \mathrm{H}\right.$ left, ${ }^{13} \mathrm{C}$ right). We remark that the resonant regions would only lie within the shaded regions, and the peaks all be of the same amplitude, if the DC magnetic field across the sample volume was sufficiently uniform. The data therefore indicate a field homogeneity better than $|\Delta B / B|<0.5 \%$. Pulses (AC) with a smaller sweep width may therefore be tolerated in the present configuration. Wideband AC pulses, on the other hand, facilitate simpler or miniaturized designs of the apparatus where the applied DC field is less homogeneous.

Selective spin inversion of this type should be considered a tool for maximizing the zero-field NMR signal amplitudes by preparing the combination of $I_{z}$ spin operators for which the noncommuting part with $H_{\mathrm{ZF}}$ is greatest. It is noteworthy to mention the case of molecules containing ${ }^{1} \mathrm{H}$ with ${ }^{19} \mathrm{~F}$, where the nuclear gyromagnetic ratios differ by less than 6 percent and the polarization difference between the two spin species at thermal equilibrium is extremely small - the fraction of $\left(I_{z}^{\mathrm{F}}-I_{z}^{\mathrm{H}}\right)$ in $\rho_{e q}$ for the system is $\left|\gamma_{\mathrm{H}}-\gamma_{\mathrm{F}}\right| /\left|\gamma_{\mathrm{H}}+\gamma_{\mathrm{F}}\right| \approx 0.03$. Inversion of either spin species enhances this by the reciprocal, i.e. a factor $\approx 33$. This case is illustrated in Figure 5 for a sample containing difluoroacetic acid $\left(\mathrm{CHF}_{2} \mathrm{COOH}\right)$. The resonances for ${ }^{1} \mathrm{H}$ and ${ }^{19} \mathrm{~F}$ at $\nu=64 \mathrm{kHz}$ occur respectively at approximately $B=1.5 \mathrm{mT}$ and $B=1.6 \mathrm{mT}$. In this case, due to low sample concentration, only the signals following the resonant pulses are visible above the noise and appear with the characteristic pattern of positive and negative peaks. This technique may similarly be applied to spin systems containing more than two NMR-active isotopes, e.g. a molecule containing ${ }^{1} \mathrm{H},{ }^{13} \mathrm{C}$ and ${ }^{15} \mathrm{~N}$. 


\section{Conclusion}

To summarize, our work demonstrates the use of NMR pulse sequences at millitesla fields on a ZULF spectrometer $(<0.1 \mu \mathrm{T})$, adding an increased capability for spin control, including spin-species selectivity. At present, the electromagnetic coils within the shielded chamber supply DC magnetic fields up to several millitesla, providing a means to study spin phenomena around the "crossover zone" between the regimes of dominant spin-spin coupling and Larmor precession (1-100 $\mu \mathrm{T}$ ), including relaxation dispersion[36, 37, 38] and also parahydrogen induced hyperpolarization, [5, 6, 39, 40, 41, 42, 43, 44, 45], which is strongly influenced by field-dependent level anticrossings and could be advantageously used in ZULF NMR. In future, we expect many opportunities for multidimensional experiments that correlate spin phenomena between the two regimes and make use of the large catalog of existing high-field pulsed NMR methods. With the use of stronger fields (in the region greater than $0.1 \mathrm{~T}$ ) chemical-shift selectivity may also be exploited.

\section{Acknowledgement}

This work was supported by the National Science Foundation under award CHE-1308381 and by the European Commission under the Marie Curie International Outgoing Fellowship programme (author MCDT, project FP7625054 ODMR-CHEM). Contents of the work do not reflect the views of the university or the European Commission.

\section{References}

[1] Blanchard, J. W., Ledbetter, M. P., Theis, T., Butler, M., Budker, D. and Pines, A., High-resolution zero-field NMR J-spectroscopy of aromatic compounds, J. Am. Chem. Soc. 135, 3607-3612 (2013).

[2] Emondts, M., Ledbetter, M. P., Pustelny, S., Theis, T., Patton, B., Blanchard, J. W., Butler, M., Budker, D. and Pines, A., Long-lived heteronuclear spin singlet states in liquids at a zero magnetic field, Phys. Rev. Lett. 112, 077601, (2014).

[3] Theis, T., Blanchard, J. W., Butler, M., Ledbetter, M. P., Budker, D. and Pines, A., Chemical analysis using J-coupling multiplets in zero-field NMR, Chem. Phys. Lett., 580, 160-165 (2013). 
[4] Butler, M., Ledbetter, M. P., Theis, T., Blanchard, J. W., Budker, D. and Pines, A., Multiplets at zero magnetic field: the geometry of zerofield NMR, J. Chem. Phys. 138, 184202-15 (2013).

[5] Butler, M., Kervern, G., Theis, T., Ledbetter, M. P., Ganssle, P. J., Blanchard, J. W., Budker, D. and Pines, A., Parahydrogen-induced polarization at zero magnetic field, J. Chem. Phys. 138, 234201 (2013).

[6] Theis, T., Ledbetter, M. P., Kervern, G., Blanchard, J. W., Ganssle, P. J., Butler, M., Shin, H. D., Budker, D. and Pines, A., Zero-field NMR enhanced by parahydrogen in reversible exchange, J. Am. Chem. Soc., 134, 3987-3990 (2012).

[7] Blanchard, J. W. and Budker, D., Zero- to ultralow-field NMR, eMagRes accepted manuscript (2016).

[8] Shim, J. H., Lee, S.-J., Hwang, S.-m., Yu, K.-K. and Kim, K., Twodimensional NMR spectroscopy of methanol at less than 5 microtesla, J. Magn. Reson. 246, 4-8 (2014).

[9] Liu, G., Li, X., Sun, X., Feng, J. Chaohui, Y., and Zhou, X., Ultralow field NMR spectroscopy with an atomic magnetometer near room temperature, J. Magn. Reson. 237, 158-163 (2013).

[10] Appelt, S., Häsing, F. W., Sieling, U., Gordji Nejad, A.,Glöggler, S. and Blümich, B., Paths from weak to strong coupling in NMR, Phys. Rev. A 81, 023420 (2010).

[11] Zax, D. B., Bielecki, A., Zilm, K., W., Pines, A. and Weitekamp, D., Zero-field NMR and NQR, J. Chem. Phys. 83, 4877-4905 (1985).

[12] Thayer, A. M. and Pines, A., Zero-field NMR, Acc. Chem. Res. 20, 47-53 (1987).

[13] Appelt, S., Wäckerle, G. and Mehring, M., Deviations from Berry's adiabatic geometric phase in a 131Xe nuclear gyroscope, Phys. Rev. Lett. 72, 3921-3924 (1994).

[14] Mössle, M., Han, S.-I., Myers, W. R., Lee, S.-K., Kelso, N., Hatridge, M., Pines, A. and Clarke, J., SQUID-detected microtesla MRI in the presence of metal, J. Magn. Reson. 179, 146-151 (2006). 
[15] Budker, D. and Romalis, M. V., Optical magnetometry, Nat. Phys. 3, 227-234 (2007).

[16] Bevilacqua, G., Biancalana, V., Dancheva, Y. and Moi, L., All-optical magnetometry for NMR detection in a micro-tesla field an unshielded environment, J. Magn. Reson. 201, 222-229 (2009).

[17] Ledbetter, M. P., Crawford, C. W., Pines, A., Wemmer, D. E., Knappe, S., Kitching, J. and Budker, D., Optical detection of NMR J-spectra at zero magnetic field, J. Magn. Reson. 199, 25-29 (2009).

[18] Ledbetter, M. P., Theis, T., Blanchard, J. W., Ring, H., Ganssle, P. J., Appelt, S., Blumich, B., Pines, A. and Budker, D., Near-zero-field nuclear magnetic resonance, Phys. Rev. Lett., 107, 107601 (2011).

[19] Yashchuk, V. V., Granwehr, J., Kimball, D. F., Rochester, S. M., Trabesinger, A. H., Urban, J. T., Budker, D. and Pines, A., Hyperpolarized Xenon Nuclear Spins Detected by Optical Atomic Magnetometry, Phys. Rev. Lett. 93, 160801, (2004).

[20] McDermott, R., Trabesinger, A. H., Muck, M., Hahn, E., Pines, A. and Clarke, J., Liquid-state NMR and scalar couplings in micro-tesla magnetic fields, Science 295, 2247-2249 (2002).

[21] Matlachov, A. N., Volegov, P. L, Espy, M. A., George, J. S. and Kraus, R. H., SQUID detected NMR in microtesla magnetic fields, J. Magn. Reson. 170, 1-7 (2004).

[22] Trabesinger, A. H., McDermott, R., Lee, S. K., Mueck, M., Clarke, J. and Pines, A., SQUID-detected liquid-state NMR in microtesla fields, J. Phys. Chem. A 108, 957-963 (2004).

[23] Bernarding, J., Buntkowsky, G., Macholl, S., Hartwig, S., Burghoff, M. and Trahms, L., J-coupling nuclear magnetic resonance spectroscopy of liquids in nT fields, J. Am. Chem. Soc. 128, 714-715 (2006).

[24] Burghoff, M., Hartwig, S., Trahms, L. and Bernarding, J., Nuclear magnetic resonance in the nanotesla range, Appl. Phys. Lett. 87, 054103 (2005). 
[25] Trahms, L. and Burghoff, M., NMR at very low fields, Magn. Reson. Imag. 28, 1244-1250 (2010).

[26] Jiminéz-Martinez, R., Kennedy, D. J., Rosenbluh, M., Donley, E. A., Knappe, S., Seltzer, S. J., Ring, H. L., Bajaj, V. S. and Kitching, J., Optical hyperpolarization and NMR detection of 129Xe on a microfluidic chip, Nature Commun. 5, 3908 (2014).

[27] Schwindt, P. D. D., Knappe, S., Shah, V., Hollberg, L. and Kitching, J., Chip-scale atomic magnetometer, Appl. Phys. Lett. 85, (2004).

[28] Thayer. A. M. and Pines, A., Composite pulses in zero-field NMR, $J$. Magn. Reson. 70, 518-522 (1986).

[29] Kemp-Harper, R., Styles, P. and Wimperis, S., $B_{1}$-selective pulses, $J$. Magn. Reson. 123, 230-236 (1996).

[30] Allred, J. C., Lyman, R. N., Kornack, T. W. and Romalis, M. V., High-sensitivity atomic magnetometer unaffected by spin-exchange relaxation, Phys. Rev. Lett. 89, 130801 (2002).

[31] Seltzer, S. J., Developments in alkali-metal atomic magnetometry, Ph.D. thesis, Princeton University (2008).

[32] Budker, D. and Jackson Kimball, D. F., Eds., Optical magnetometry, Cambridge University Press (2013).

[33] van Beek, J. D., Carravetta, M., Antonioli, G. C. and Levitt, M. H., Spherical tensor analysis of NMR signals, J. Chem. Phys 122, 244510 (2005).

[34] Garwood M. and DelaBarre L., The return of the frequency sweep: designing adiabatic pulses for contemporary NMR, J. Magn. Reson. 153, 155-177 (2001).

[35] Garwood M. and Merkle, H., Heteronuclear spectral editing with adiabatic pulses, J. Magn. Reson. 94, 180-185 (1991).

[36] Ganssle, P. J., Shin, H. D., Seltzer, S. J., Bajaj, V. S., Ledbetter, M. P., Budker, D., Knappe, S., Kitching, J. and Pines, A., Ultra-low-field NMR relaxation and diffusion measurements using an optical magnetometer, Angew. Chem. Int. Edn. 53, 9766-9770 (2014). 
[37] Steele, R. M., Korb, J.-P., Ferrante, G. and Bubici, S., New applications and perspectives of fast field cycling NMR relaxometry, Magn. Reson. Chem. early view online, DOI: 10.1002/mrc.4220 (2015).

[38] Hartwig, S., Voigt, J., Scheer, H. J., Albrecht, H. H., Burghoff, M. and Trahms, L., Nuclear magnetic relaxation in water revisited, J. Chem. Phys. 135, 054201 (2011).

[39] Grant, A. K. and Vinogradov, E., Development of a fast field-cycling method for polarizing C13 using parahydrogen-induced polarization, Proc. Int. Soc. Magn. Reson. Med, 17, 2449 (2015).

[40] Colell, J., Türschmann, P., Glöggler, S., Schlecker, P., Theis, T., Ledbetter, M. P., Budker, D., Pines, A., Blümich, B. and Appelt, S., Fundamental aspects of parahydrogen enhanced low-field nuclear magnetic resonance, Phys. Rev. Lett., 110, 137602 (2013).

[41] Theis, T., Ganssle, P., Kervern, G., Knappe, S., Kitching, J., Ledbetter, M. P., Budker, D. and Pines, A., Parahydrogen enhanced zero-field nuclear magnetic resonance, Nat. Phys., 7, 571-575 (2011).

[42] Theis, T., Truong, M., Coffrey, A., Shchepin, R., Waddell, K., Shi, F., Goodson, B., Warren, W. S., and Chekmenev, E., Microtesla SABRE enables $10 \%$ nitrogen-15 nuclear spin polarization, J. Am. Chem. Soc., 137, 1404-1407 (2015).

[43] Truong, M., Theis, T., Coffrey, A., Shchepin, R., Waddell, K., Shi, F., Goodson, B., Warren, W. S., and Chekmenev, E., 15N hyperpolarization by reversible exchange using SABRE-SHEATH, J. Phys. Chem. C, 119, 8786-8797 (2015).

[44] Mewis, R. E., Green, R. A., Cockett, M. C. R., Cowley, M. J., Duckett, S. B., Green, G. G. R., John, R. O., Rayner, P. J. and Williamson, D. C., Strategies for the hyperpolarization of acetonitrile and related ligands by SABRE, J. Phys. Chem. B, 119, 1416-1424 (2015).

[45] Pravdivtsev, A. N., Yurkovskaya, A. V., Zimmerman, H., Vieth, H.M. and Ivanov, K. L., Transfer of SABRE-derived hyperpolarization to spin-1/2 nuclei, RSC Adv., 5, 63615-63623 (2015). 

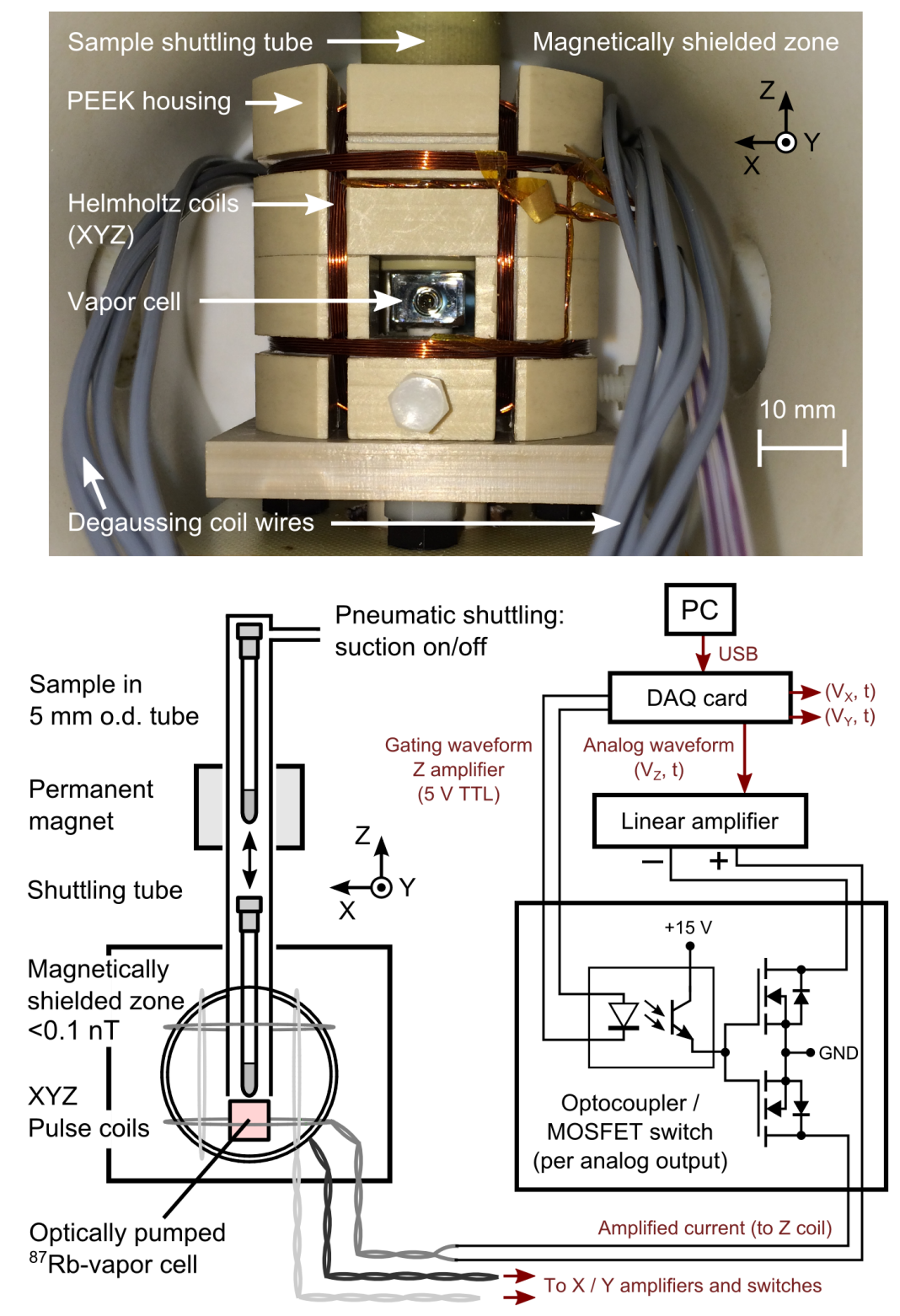

Figure 1: Components of the low-field NMR instrument, based on a ${ }^{87} \mathrm{Rb}$ atomic magnetometer. 

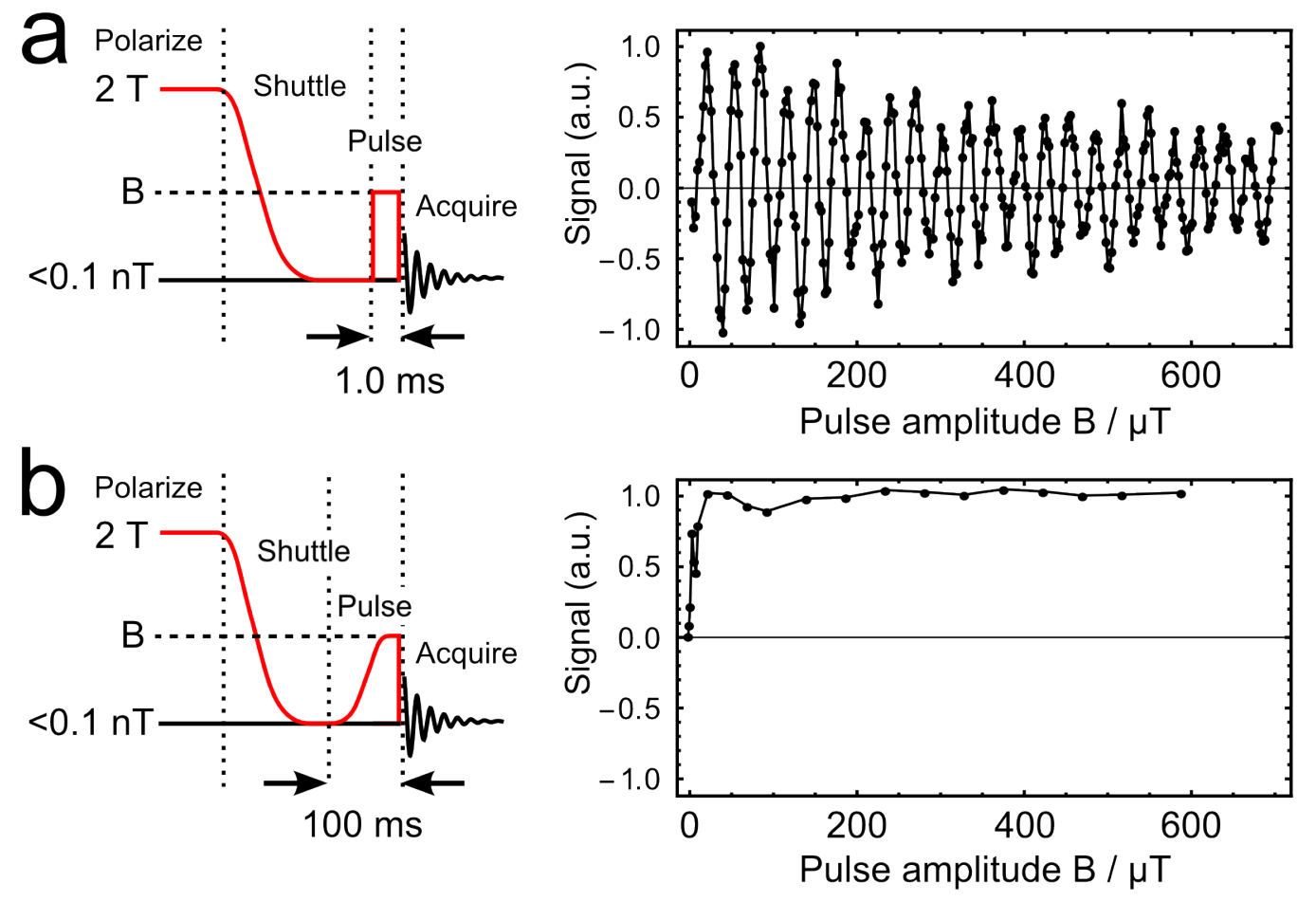

Figure 2: Amplitudes of the zero-field NMR signal for $\left[{ }^{13} \mathrm{C}\right]$-formic acid $(0.1 \mathrm{~mL}, 99 \%)$ following prepolarization at $2 \mathrm{~T}$ and adiabatic transport into zero field then application of magnetic fields along the $z$ axis: (a) a square pulse of duration $1.0 \mathrm{~ms}$; (b) a hyperbolic secant pulse of duration $100 \mathrm{~ms}$, for adiabatic remagnetization. The data in the plots share the same normalization: 1 a.u. $\approx 200 \mathrm{fT}$. 

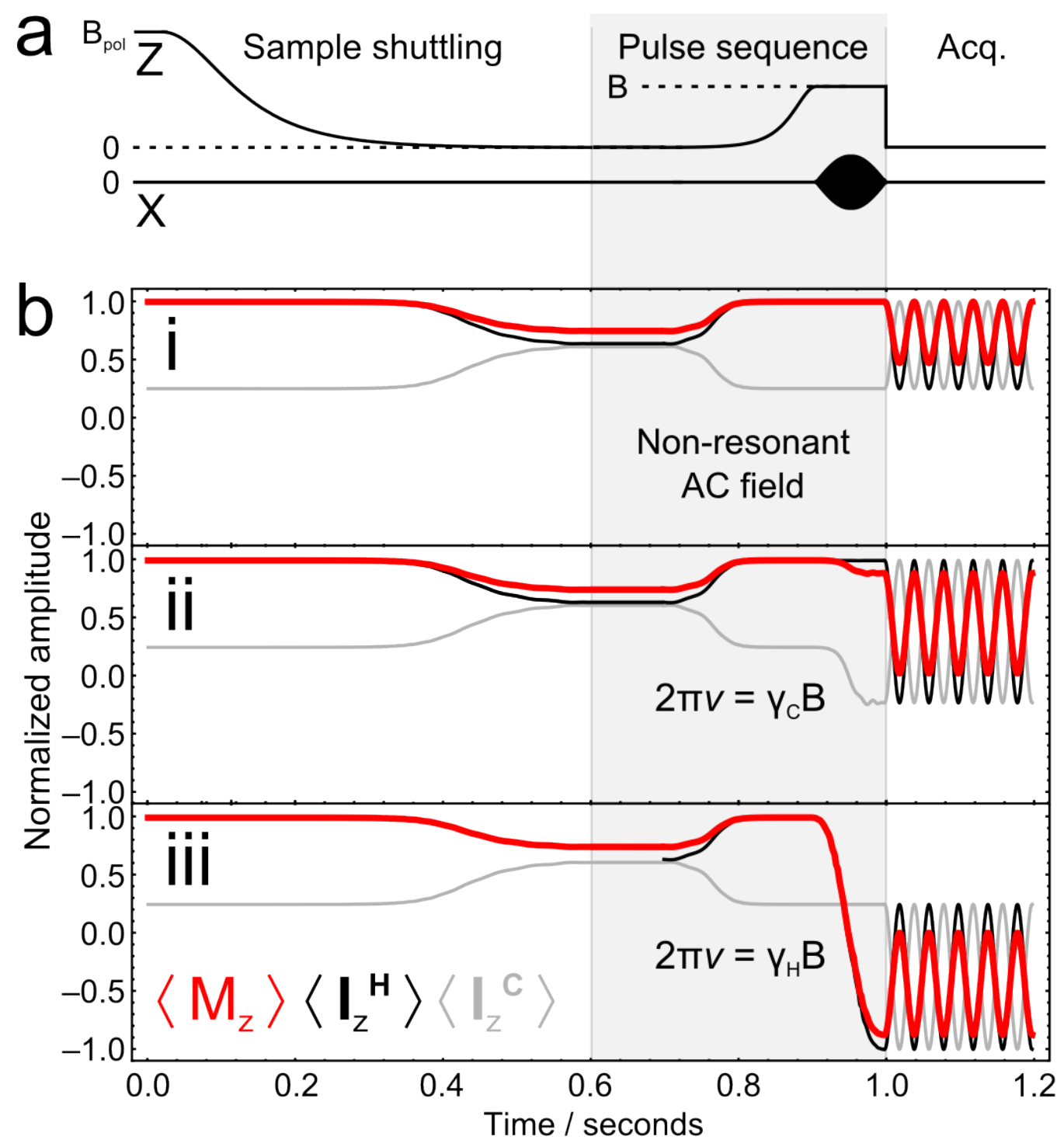

Figure 3: Spin-selective adiabatic inversion in a zero-field NMR experiment: (a) $x$-axis and $z$-axis projections of the magnetic field at the NMR sample (vertical axis not to scale), starting with the prepolarization field $B_{\text {pol }}$; (b) Simulated trajectories of the total magnetization $\left\langle M_{z}\right\rangle=\gamma_{\mathrm{H}}\left\langle I_{z}^{\mathrm{H}}\right\rangle+\gamma_{\mathrm{C}}\left\langle I_{z}^{\mathrm{C}}\right\rangle$ and the spin polarizations $\left\langle I_{z}^{\mathrm{H}}\right\rangle$ and $\left\langle I_{z}^{\mathrm{C}}\right\rangle$ for a ${ }^{1} \mathrm{H}^{-13} \mathrm{C}$ pair using parameters $J_{\mathrm{CH}}=25 \mathrm{~Hz}, \Delta \nu=400 \mathrm{~Hz}$ and $\nu=4000 \mathrm{~Hz}$. The duration of the swept-frequency AC pulse is $100 \mathrm{~ms}$. The plots illustrate how the signal amplitude and phase depends on whether the AC pulse is (i) non-resonant, or resonant with the (ii) ${ }^{13} \mathrm{C}$ or (iii) ${ }^{1} \mathrm{H}$ spin precession. 


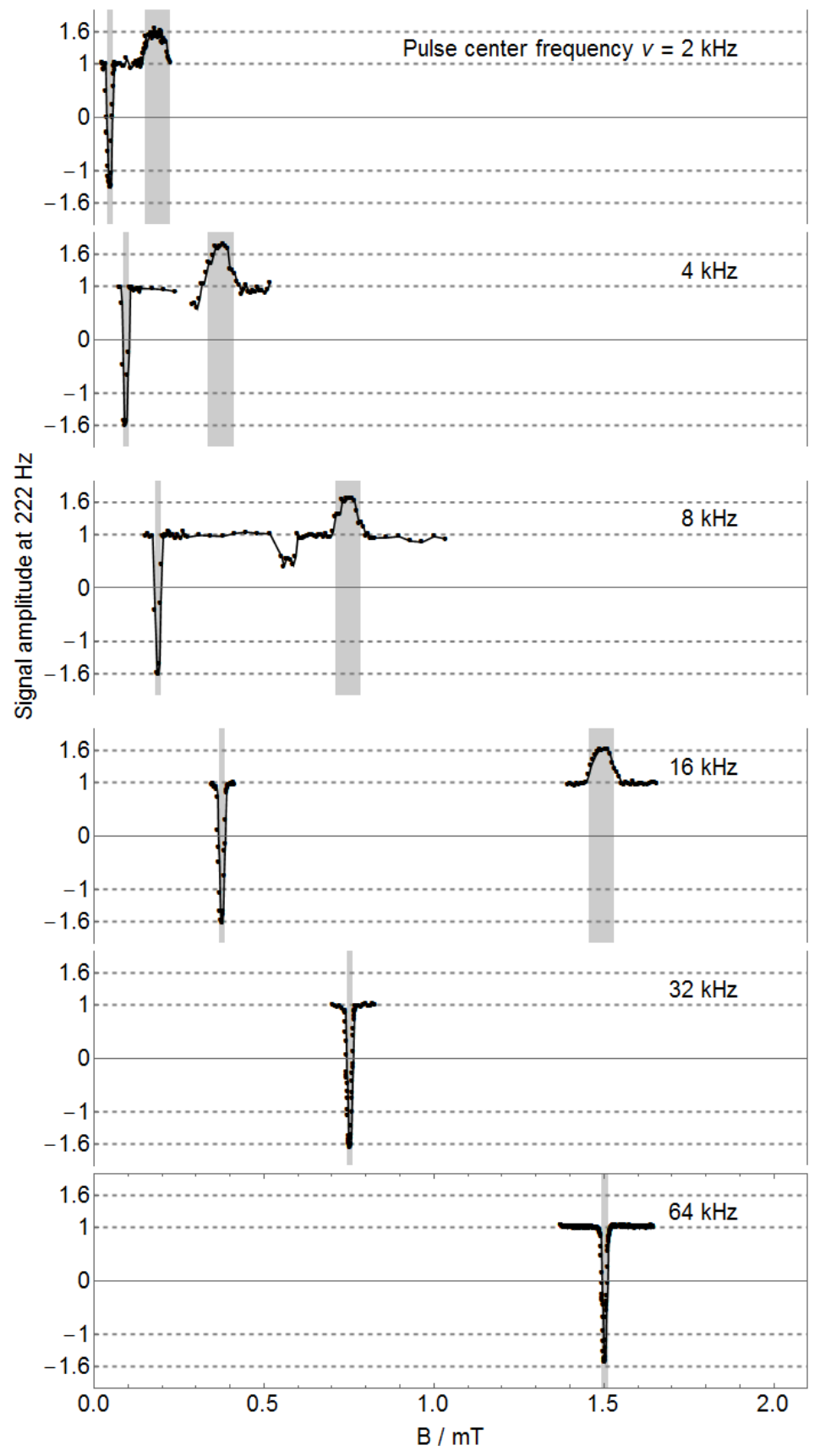

Figure 4: Amplitudes of the zero-field NMR signal for $\left[1-{ }^{13} \mathrm{C}\right]$-formic acid obtained after a swept-frequency pulse vs. center frequency $\nu$ and strength of the DC field $B$ as depicted in Figure 3a. Vertical shaded bands represent the predicted range of resonant fields $\left({ }^{1} \mathrm{H}\right.$ narrow, ${ }^{13} \mathrm{C}$ wide) given the sweep width of the $\mathrm{AC}$ pulse $\Delta \nu=800 \mathrm{~Hz}$. The vertical scale is normalized to the intensity of the signal with an off-resonance AC pulse. The dotted horizontal lines at $\pm\left(\gamma_{\mathrm{H}}+\gamma_{\mathrm{C}}\right) /\left(\gamma_{\mathrm{H}}-\gamma_{\mathrm{C}}\right) \approx \$ 5.6$ represent the maximum achievable signal. 


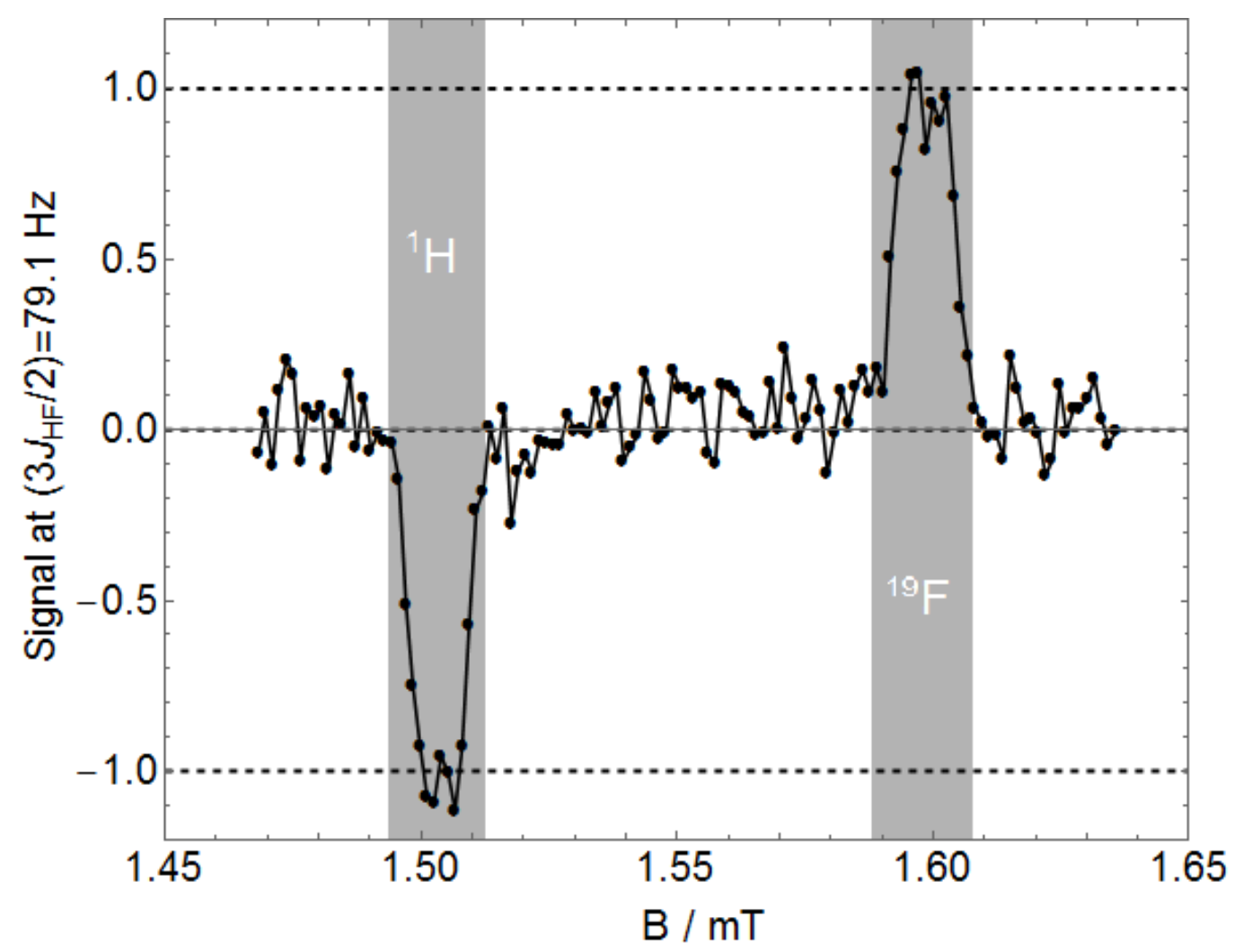

Figure 5: Amplitudes of the zero-field NMR signal for difluoroacetic acid $\left(\mathrm{CHF}_{2} \mathrm{COOH}\right.$, spectral peak at $\left.(3 / 2) J_{\mathrm{HF}}=79.1 \mathrm{~Hz}\right)$ vs. the DC field $B$ after a swept-frequency pulse with $\nu=64 \mathrm{kHz}, \Delta \nu=800 \mathrm{~Hz}$. The shaded zones represent the expected range of resonant fields. 
Adiabatic swept-frequency inversion pulse $(16 \pm 0.4) \mathrm{kHz}$

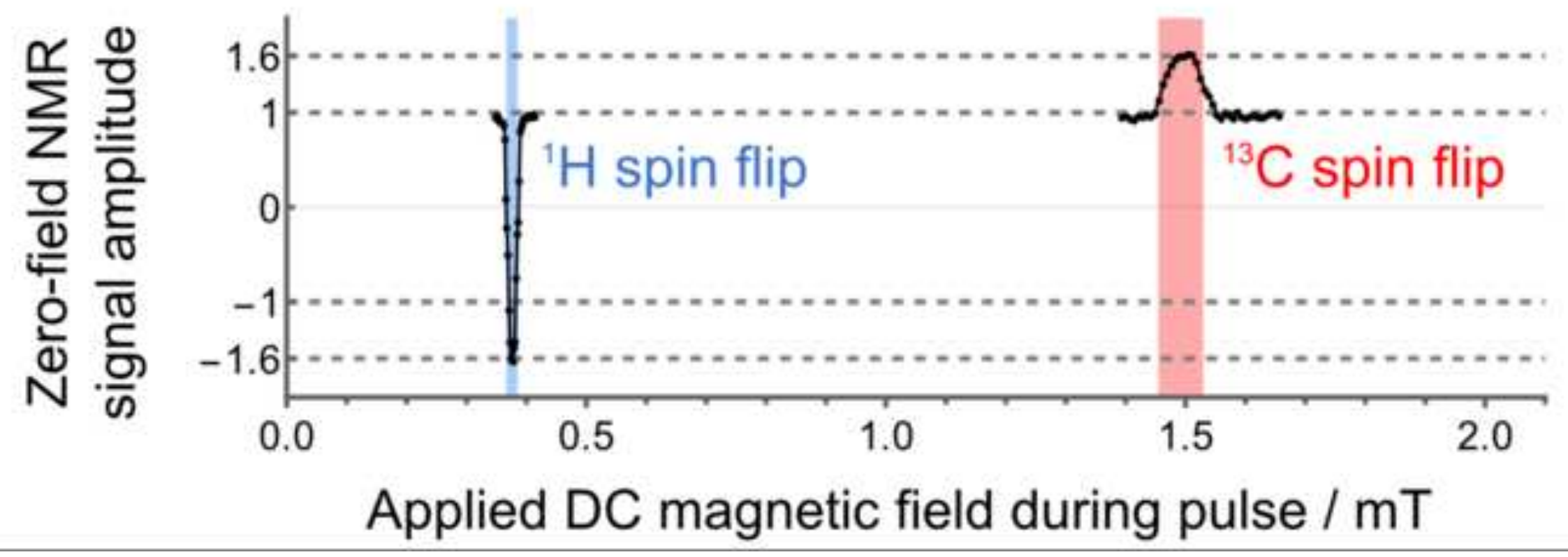

